\title{
Microstructural Properties and Defect Evolution on Nitride Compound Semiconductors Grown on Patterned Substrates: A Transmission Electron Microscopy Study
}

\author{
Y.H. Kim, ${ }^{*}$ Y.K. Noh*****, S.H. Han***, M.D. Kim***
}

*Korea Research Institute of Standards and Science, 1 Doryong-Dong, Yuseong-Gu, Daejeon 305340 , Republic of Korea

**Wooree LST Corporation, Ansan-shi, Kyungki-do 425-833, Republic of Korea

***Department of Physics, Chungnam National University, 220 Gung-Dong, Yuseong-Gu, Daejeon 305-764, Republic of Korea

Group III-nitrides compound semiconductors have been recognized to be a great variety of applications such as UV photodetectors, laser diodes, and high-speed field-effect transistors due to their direct band gap, emissive property, and extremely large heterojunction offsets. Recently, growing nitride compound semiconductors on a patterned substrates (PSs), such as a sapphire and a silicon, without a mask or interruption has recently been considered as an alternative way to reduce the threading dislocations and enhance the light output power as well as the internal quantum efficiency of light reflections through geometrical effects [1-3]. However, most studies related to PS have focused on evaluating the device's capability and the reduction of dislocation density. Until recently, the growth mechanism and the defect evolution for nitride compound semiconductors grown on a dot-patterned substrate have not been widely reported. In this presentation, we report on the growth behavior and the microstructural properties of thin nitride-layer grown on a PS from a microstructural point of view.

Nitride compound semiconductors were grown on a plane and a patterned substrates using metalorganic chemical vapor deposition (MOCVD) and molecular beam epitaxy (MBE) methods. The substrates were patterned by dry etching to form a protruding pattern array. After the fabrication of the pattern array, we noted that the pattern was uniform in size and arranged well. Bright-field TEM (BFTEM) images, selected-area electron diffraction (SAED) patterns, high-resolution TEM micrographs (HRTEM), and high-angle annular dark-field (HAADF) images were collected by fieldemission gun (S)TEM operating $300 \mathrm{kV}$. Specifically, the detailed interface structures between the nitrides and the PSs were studied using TEM techniques

Figure 1 shows the cross-sectional BFTEM images, taken using multi-beam diffraction conditions, for the GaN structures grown on patterned sapphire substrate. The thickness of the GaN layer in Fig. 1(b) is larger than that of the GaN structure in Fig. 1(a). A discontinuous surface and several grains were observed in Fig. 1(a). The grains I, II, III, and IV had different crystallographic orientations. In addition, a few vacant spaces were detected among the grains. On the other hand, a continuous surface and a dominant single grain were observed in Fig. 1(b), although a few small minor grains existed near the protruding patterns. The dislocation density was low in the GaN layer (Fig. 1(b)). In Figs. 1(a) and 1(b), the morphology and microstructural properties of the GaN structures were determined by the difference in the growth temperature. Specifically, the detailed control of growth parameters were required to achieve a high-quality epitaxial layer on patterned substrates because the nucleation and the growth of materials were affected by the protruding patterns and the surface state by unintended treatment during the dry etching process.

We have also studied the growth of nitride compound semiconductors on pa terned silicon substrates. The dependence on the geometrical shape and the size of the protruding patterns of 
nitride structures is introduced when they were grown on a patterned silicon substrate. Finally, we demonstrate the growth mechanism and the propagation behaviors of various defects, based on our transmission electron microscopy study.

\section{References}

[1] H. Gao, F. Yan, Y. Zhang, J. Li, Y. Zeng, and G. Wang, J. Appl. Phys. 103, 014314 (2008)

[2] 13. J. Mei, F.A. Ponce, R.S. Qhalid Fareed, J.W. Yang, and M. Asif Khan, Appl. Phys. Lett. 90, 221909 (2007).

[3] 16. C.H. Chiu, H.H. Yen, C.L. Chao, Z.Y .Li, P. Yu, H.C. Kuo, T.C. Lu, S.C. Wang, K.M. Lau, and S.J. Cheng, Appl. Phys. Lett. 93, 081108 (2008)

[4] This research was supported by Future-based Technology Development Program through the National Research Foundation of Korea (NRF) funded by the Ministry of Education, Science and Technology (Grant No. 20100029332), and in part by Korea Research Council of Fundamental Science and Technology (KRCF) through the KRISS project of " Development of Characterization Techniques for Nano-materials Safety".

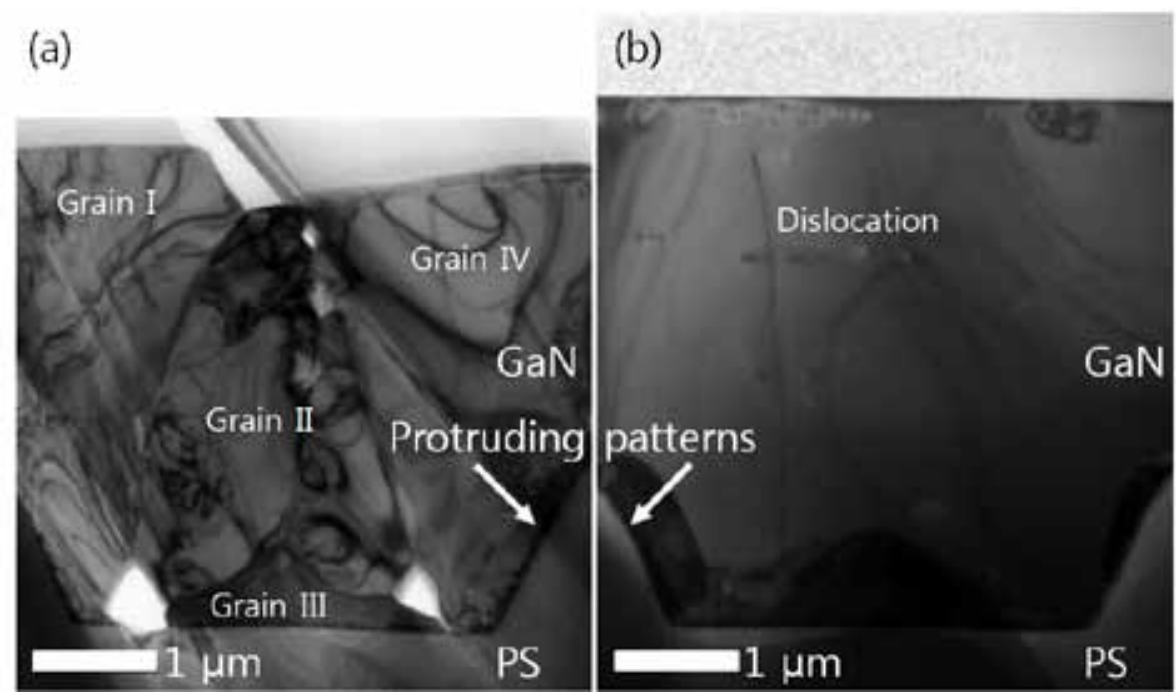

FIG. 1. Cross-sectional BFTEM images of the GaN structures grown on pa tterned sapphire substrates. (a) High growth temperature. (b) Low growth temperature. 